\title{
Optical design and laboratory characterisation of CAWS: the Calibration and Alignment Wavefront Sensor for high-order adaptive optics
}

\author{
Nicolás Dubost ${ }^{\mathrm{a}}$, Nazim Ali Bharmal ${ }^{\mathrm{a}}$, Marc Dubbeldamª ${ }^{\mathrm{a}}$ Daniel Hölck ${ }^{\mathrm{a}}$, and Richard M \\ Myers $^{\mathrm{a}}$ \\ ${ }^{a}$ CfAI, Department of Physics, Durham University, South Road, Durham DH1 3LE, United \\ Kingdom
}

\begin{abstract}
The Calibration and Alignment Wavefront Sensor (CAWS) is a common-path interferometer for high-order adaptive optics (AO). With a resolution comparable to that of a 51-by-51 sub-apertures Shack-Hartmann sensor (SHS), it can directly measure the phase at a pupil without explicit reconstruction. In the past, interferometers have been deemed unsuitable for AO. Using a separate reference beam meant the interference pattern was susceptible to piston variations. Unlike SH wavefront sensors, interferometers are usually monochromatic, meaning they can only use a small portion of light. This is solved by CAWS' common-path design in which the reference beam is self-generated using a share of the input light. As a consequence, the reference beam always has the same piston as the measured wavefront and has the same intensity. This holds true for all wavelengths and allows CAWS to do interferometry with narrow-band polychromatic light. Having more flux than monochromatic interferometers, CAWS can run at a higher frame rate. Empirical tests show the instrument's frequency response is kept in the linear regime at low RMS phase aberrations. Both these features allow CAWS to measure, in real-time, residual phase aberrations in closed-loop AO systems. An application of this is to distinguish and discard speckles from small features in high-order or extreme AO. This work presents the optical design and characterisation of the instrument using laboratory results. The results show the instrument's frequency response in a monochromatic regime.
\end{abstract}

Keywords: adaptive optics, high-order, interferometry, wavefront sensor, chough

\section{INTRODUCTION}

In order to meet the challenges of direct exoplanets imaging, different techniques have been proposed for the the next generation of extremely large telescopes. Amongst the most promising, coronagraphs ${ }^{1}$ and apodised pupils? suffer from speckles in the point spread function (PSF). Attenuating the speckles can lead to enhancing the image's dynamic range by a factor of $10^{4} .^{2}$ Atmospheric speckles are short-lived and easily averaged out, whereas quasi-static speckles, being generally more chromatic and long-lived cannot be attenuated through averaging or differential techniques like simultaneous spectral differential imaging (SSDI). ${ }^{3}$

The main source of quasi-static speckles is the presence of non-common path aberrations (NCPA) between the science camera and the wavefront sensor (WFS). In order to correct for most of the NCPAs, extreme adaptive optics (XAO) systems use post-AO wavefront sensing, placing a lower-bandwidth sensor of higher sensitivity between the $\mathrm{AO}$ system and the coronagraph. Examples of this are the Gemini Planet Imager ${ }^{4}$ and more recently the VLT-SPHERE with the use of the Zernike sensor ZELDA. ${ }^{5}$

This paper presents the laboratory work done for a novel field-modulation point-diffraction interferometer (FM/PDI), called the Calibration and Alignment Wavefront Sensor (CAWS). ${ }^{6}$ The basic scheme of CAWS is presented in Fig. 1. In Plane A, at the entrance pupil, a small-angle beam splitter divides the light into modes.

Further author information: (Send correspondence to N.D.)

N.D.: E-mail: nicolas.s.dubost@durham.ac.uk, Telephone: +44 (0)191 3343485

N.A.B.: E-mail: n.a.bharmal@durham.ac.uk, Telephone: +44 (0)191 3341409 


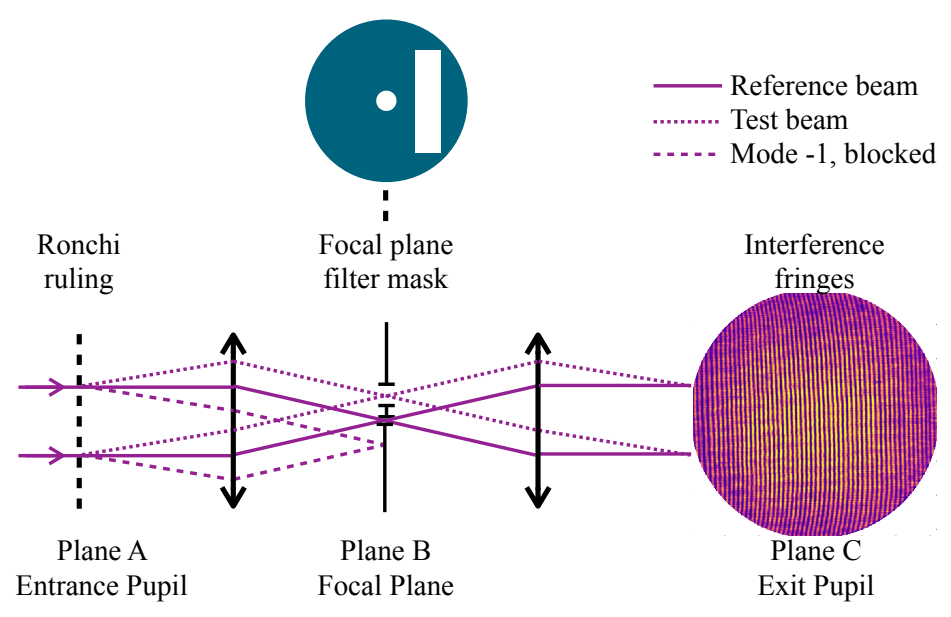

Figure 1. Basic illustration of the CAWS concept.

The beam splitter is in this case a Ronchi ruling acting as a transmission grating. In the focal plane a filter mask containing two apertures, a small pinhole and a larger (in this case rectangular) aperture. The central mode (i.e. mode 0) is filtered by the pinhole letting through only the core of the PSF. Once re-collimated, this will produce a flat wavefront which constitutes the interferometer's reference beam. The test beam, on the other hand, is produced by letting mode +1 through the second, bigger aperture. The beams later interfere in the exit pupil producing an interferogram from which both amplitude and phase can be retrieved. This interferometer is said to be common-path because the reference and the test beams do not divide into separate arms, but instead travel through the same optics. As a consequence CAWS is compact and very robust against vibrations and local turbulence.

\section{INTERFEROMETRY AND WAVEFRONT RECONSTRUCTION}

CAWS codes both the amplitude and the phase of an electric field at the entrance pupil into an interferogram at the exit pupil. The formalism is described in a previous publication. ${ }^{6}$ Right before reaching Plane A, the electric field is

$$
\Psi_{0}=P e^{i \varphi}=P_{0}(1-\epsilon) e^{i \varphi},
$$

where $\varphi$ is the phase, $P$ is the amplitude, $P_{0}$ is its average across the pupil and $\epsilon$ is a zero-mean function describing a local scintillation. Since in most astronomical AO applications $P=P_{0}$, the model can be simplified to only describe a modulation of the phase. In this case, the intensity of the interferogram in Plane $\mathrm{C}$ is given by

$$
I_{C}=b^{2}+\frac{1}{\pi^{2}} P_{0}^{2}+\frac{2 b}{\pi} P_{0} \cos \left(\frac{2 \pi}{T} x-\varphi\right),
$$

where $T$ is the thickness of a line-pair in the grating and $b$ is the amplitude of the reference beam. The fringes in the interferogram are represented by the cosine term in Eq. 2 and are an image of the fringes. This image is modulated by the phase $\varphi$. The wavefront reconstruction algorithm relies mostly on Fourier transforms, making it very fast and scalable. For this reason CAWS can provide fast high-resolution wavefront sensing for high-order and extreme $\mathrm{AO}$ systems.

\section{OPTICAL DESIGN}

The Ronchi ruling placed in Plane A fits 52 line-pairs into the pupil and acts as a diffraction grating producing dispersion modes. This gives CAWS a resolution comparable to that of a 51-by-51 SHS. The light coming from the pupil is later focused and collimated once again by a 4 -f system, guaranteeing telecentricity.

At the focal plane the light is filtered by a mask as shown in Fig. 2. Four masks were manufactured. In two 


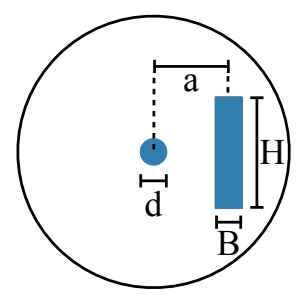

Figure 2. Diagram of the focal plane filter mask.

of them the diameter of the central pinhole is $d=2.5 \lambda / D$ and in the two others $d=2.2 \lambda / D$, where $D$ is the diameter of the entrance pupil. A flatter reference beam could be achieved by reducing $d$, but this would also increase the difficulty in aligning the filter mask resulting in a faint reference beam and in low fringe visibility. We were successful in aligning both masks achieving good visibility and are confident in being able to align smaller pinholes with a diameter $d=\lambda / D$.

Two masks have square apertures for mode +1 , where $H=B$. In this case CAWS' resolution is the same in the $X$ direction and in the $Y$ (vertical) direction. In the other two masks $H=6 B$. On the one hand, using this configuration lets through more vertical frequencies, increasing the vertical resolution in the $Y$ direction by a factor of 6 . As a result, CAWS has the resolution of a 51-by-311 SHS. On the other hand, this allows for less filtering and greater noise. This paper only presents results using the low-noise configuration with $H=B$, as a higher resolution was not required at this initial stage. A comparative study between the two configurations is still to be done.

The distance of mode +1 with respect to the centre of the pinhole is

$$
a=f_{1} \lambda_{0} / T
$$

where $f_{1}$ is the effective focal length of the lens producing the focal plane and $\lambda_{0}$ is the central wavelength around which the instrument is designed and optimised. This distance is the same for all masks as $\lambda_{0}=675 \mathrm{~nm}$ and $T=0.2 \mathrm{~mm}$. As a result $B$ is also a constant as $B=2 a / 3$ in order to avoid aliasing. In turn, the maximum bandwidth of the instrument is

$$
\Delta \lambda=B \lambda_{0} / a=2 \lambda_{0} / 3 .
$$

The experiments presented here were all carried out with a monochromatic source of light. Polychromatic effects will be studied in a further paper.

All masks are presented in Table 1 and an image of Mask 1, the mask used for all results presented in this paper, is shown in Fig. 3.

Table 1. Dimensions of focal plane filter masks in $\mu \mathrm{m}$

\begin{tabular}{|l|llll|}
\hline & Mask 1 & Mask 2 & Mask 3 & Mask4 \\
\hline d & 14 & 14 & 16 & 16 \\
a & 338 & 338 & 338 & 338 \\
B & 225 & 225 & 225 & 225 \\
H & 225 & 1350 & 225 & 1350 \\
\hline
\end{tabular}

\section{LABORATORY CHARACTERISATION}

CAWS has been designed to be integrated with CHOUGH, a high-order upgrade for CANARY ${ }^{7}$ and shown in Fig. 4. CHOUGH provides a 31-by-31 SHS in closed-loop with a 32-by-32 Boston Micromachines Corporation Kilo-DM. CHOUGH can also support an imager for PSF measurements. 


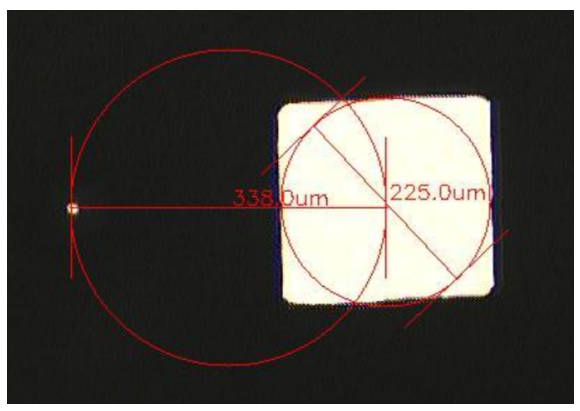

Figure 3. Image of Mask 1.

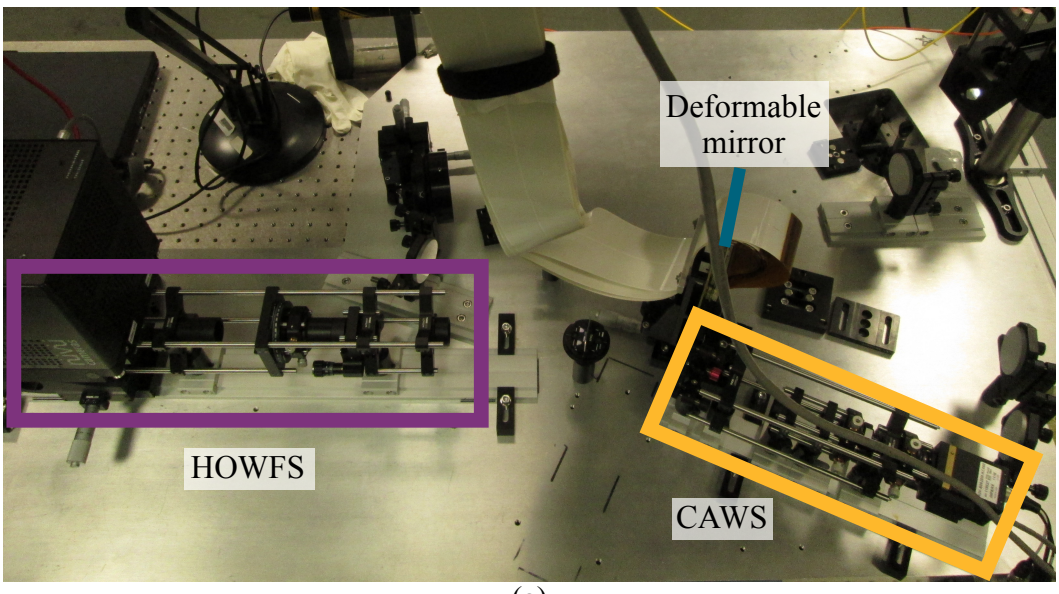

(a)

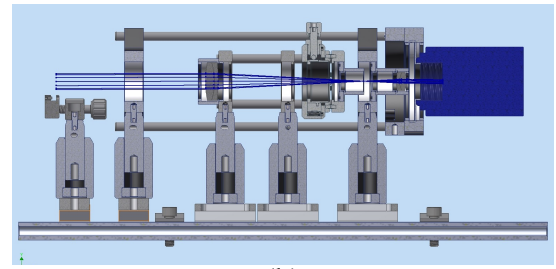

(b)

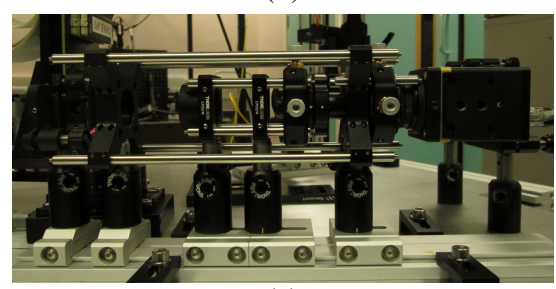

(c)

Figure 4. (a) Image of CHOUGH, its own Shack-Hartmann High-Order WFS (HOWFS) and CAWS. (b) Mechanical model of CAWS. (c) Later view of CAWS.

\subsection{Frequency and amplitude response}

In this setup a beam-splitter feeds the beam to the high-order WFS (HOWFS) and to CAWS after the deformable mirror (DM). Since both WFSs can measure the aberrations introduced by the DM, the reconstructed wavefronts can be compared in order to use the HOWFS as a benchmark for CAWS.

In order to measure CAWS' transfer function, pure tones of increasing frequency are produced by the DM. The pure tones are sinusoidal wavefronts, perpendicular to the fringes and in the form

$$
t(x, y)=A \cos (2 \pi \kappa y)
$$

where $\kappa$ is the spatial frequency of the tone and $A$ is the amplitude. Furthermore, in order to test the linearity of the response, transfer functions for multiple amplitudes $A$ are tested. The same measurements are carried out with HOWFS, a well known SHS to serve as calibration. By computing the ratio between the transfer functions, the non-linearities of the DM are discarded. Both sets of measurements and the ratio between them are shown in Fig. 5.

In both Fig. 5(a) and Fig. 5(b) the measured amplitude decreases as the frequency increases, given a constant DM command amplitude. This is attributed to non-linearities in the DM as they are cancelled out when computing the ratio between the measurements. Indeed, there is a flat region in Fig. 5(c) in which CAWS' transfer function behaves the same as HOWFS'. The flat region is CAWS' linear regime. 


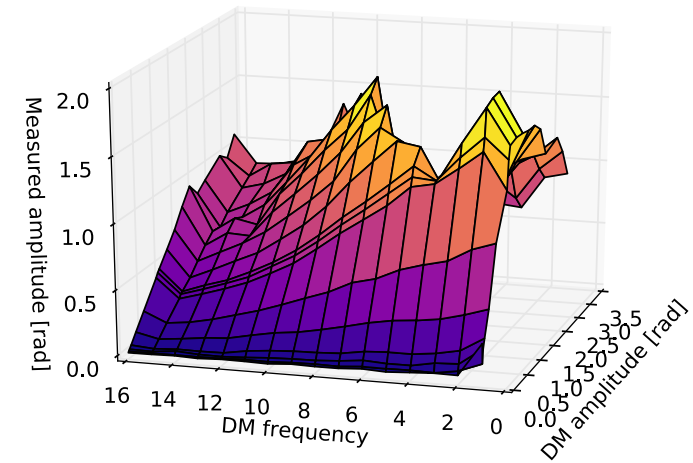

(a)

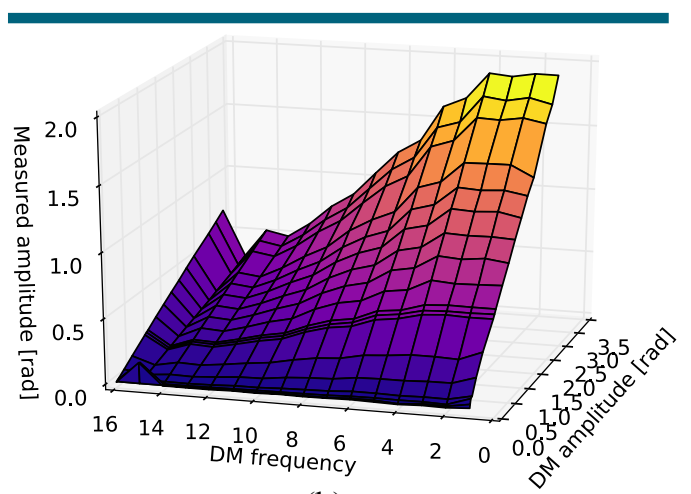

(b)

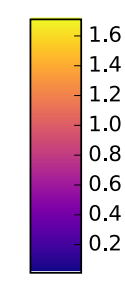

CAWS

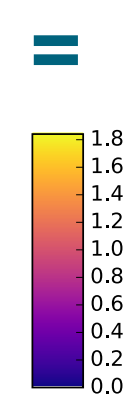

HOWFS

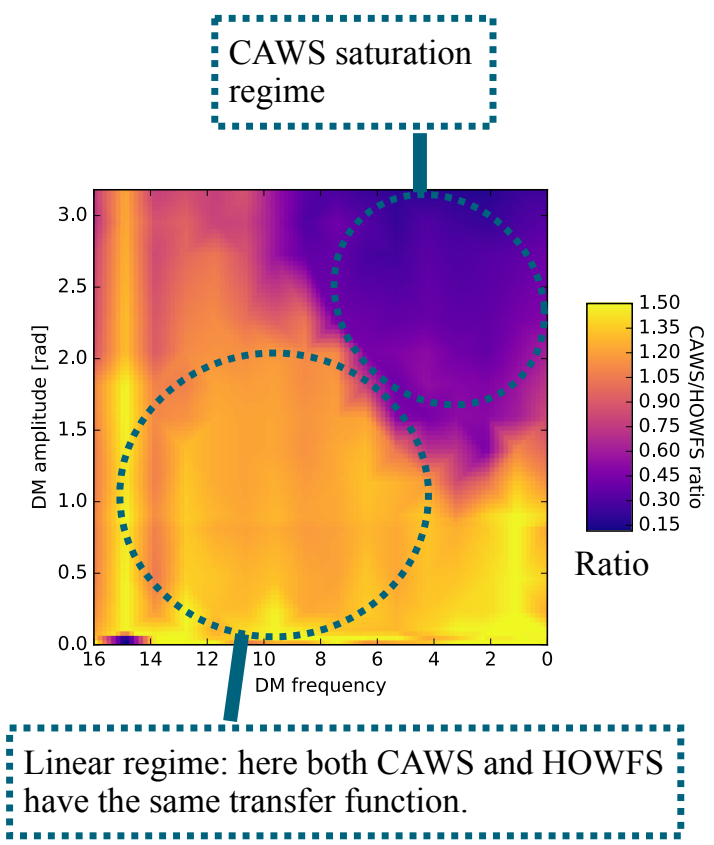

(c)

Figure 5. Measured wavefront amplitude as a function of DM command amplitude and spatial frequency with a sinusoidal input for (a) CAWS and (b) HOFWS. (c) Ratio between (a) and (b).

\section{CONCLUSION AND PROSPECTS}

Further tests will be performed, with the aim of testing wavefront reconstruction speed, chromatic effects and integration to an AO control loop. For this purpose two major tasks need to be achieved. The first is to produce all the calibration and interface algorithms between CAWS and DARC, ${ }^{8}$ the real-time computer controlling CHOUGH. This will include computing a linear transformation between CAWS data and SHS data. The second is to build a multimode illumination unit, capable of providing multiple wavelengths and bandwidths.

Once this is done, the next step is to pursue the two major goals CAWS was conceived to address. The first one is the reduction of quasi-static speckles due to NCPAs in coronagraphic AO. The other one is to measure piston differentials between disconnected pupils. Preliminary simulations are encouraging, showing good sensitivity to piston differences when using a small pinhole $(d \simeq \lambda / D)$. This could both solve the island problem produced by telescope spiders and the co-phasing problem for segmented mirrors.

\section{ACKNOWLEDGMENTS}

We acknowledge CONICYT Programa Formacion de Capital Humano Avanzado 72160371, and Science and Technology Facilities Council Project Research Development funding (ST/L002213/1) for CHOUGH.

\section{REFERENCES}

[1] Rouan, D., Riaud, P., Boccaletti, A., Clénet, Y., and Labeyrie, A., "The four quadrant phase mask coronagraph. I. Principle," Pasp 112(777), 1479-1486 (2000).

[2] Marois, C., Doyon, R., Racine, R., and Nadeau, D., "Efficient Speckle Noise Attenuation in Faint Companion Imaging," Publications of the Astronomical Society of the Pacific 112(767), 91-96 (2000). 
[3] Macintosh, B. A., Graham, J. R., Palmer, D. W., Doyon, R., Dunn, J., Gavel, D. T., Larkin, J., Oppenheimer, B., Saddlemyer, L., Sivaramakrishnan, A., Wallace, J. K., Bauman, B., Erickson, D. A., Marois, C., Poyneer, L. A., and Soummer, R., "The Gemini Planet Imager: from science to design to construction," Adaptive Optics Systems. Edited by Hubin 7015(2008), 701518 (2008).

[4] Macintosh, B., Graham, J., Palmer, D., Doyon, R., Gavel, D., Larkin, J., Oppenheimer, B., Saddlemyer, L., Wallace, J. K., Bauman, B., Evans, J., Erikson, D., Morzinski, K., Phillion, D., Poyneer, L., Sivaramakrishnan, A., Soummer, R., Thibault, S., and Veran, J.-P., "The Gemini Planet Imager," American Astronomical Society 39(2006), 62720L-62720L-12 (2006).

[5] N'Diaye, M., Vigan, A., Dohlen, K., Sauvage, J.-F., Caillat, A., Costille, A., Girard, J. H. V., Beuzit, J.-L., Fusco, T., Blanchard, P., Le Merrer, J., Le Mignant, D., Madec, F., Moreaux, G., Mouillet, D., Puget, P., and Zins, G., "ZELDA, a Zernike wavefront sensor for the fine measurement of quasi-static aberrations in coronagraphic systems: concept studies and results with VLT/SPHERE," 99096S (2016).

[6] Bharmal, N. A., Myers, R. M., Basden, A. G., and Reeves, A. P., "An interferometric wavefront sensor for high-sensitivity low-amplitude measurements," 84472J-84472J-13 (2012).

[7] Myers, R. M., Hubert, Z., Morris, T. J., Gendron, E., Dipper, N. a., Kellerer, A., Goodsell, S. J., Rousset, G., Younger, E., Marteaud, M., Basden, A. G., Chemla, F., Guzman, C. D., Fusco, T., Geng, D., Le Roux, B., Harrison, M. a., Longmore, A. J., Young, L. K., Vidal, F., and Greenaway, A. H., "<title>CANARY: the on-sky NGS/LGS MOAO demonstrator for EAGLE</title>," $7015(2008), 70150 \mathrm{E}-70150 \mathrm{E}-9$ (2008).

[8] Basden, A. G. and Myers, R. M., "The Durham adaptive optics real-time controller: Capability and Extremely Large Telescope suitability," Monthly Notices of the Royal Astronomical Society 424(2), 1483-1494 (2012). 